\title{
Magnetic structures in the solar atmosphere
}

\author{
MurRay D. SCIFFER
}

Linear and nonlinear solutions for two-dimensional magnetic arcades $\left(\frac{\partial}{\partial z}=0\right)$ using a Grad-Shafranov equation in which the axial magnetic field and the pressure are specified as functions of the component of the vector potential in the $z$ direction are re-examined.

We first consider the situation where the magnetic field has a bipolar structure on the iower (pnocospneric) boundary. To compuie nonlinear soluiluns, une is resiricied iu seeking solutions on a finite computational domain with specified boundary conditions. We consider three basic models which have appeared in the literature and examine in detail the behaviour of neutral lines in these models. The first model has boundary conditions which restrict the magnetic flux to the computational domain. This is achieved via the use of Dirichlet boundary conditions. In another model the field is laterally restricted by means of Dirichlet boundary conditions and free to extend vertically by means of a Neumann condition at the top of the domain. For such fields, bifurcating solutions only appear for a narrow range of values for the parameter $\lambda$ (the ratio of a typical length scale of the field to the gravitational scale height). Nevertheless, we show that the presence of this parameter is essential for bifurcating solutions in such domains. For the third model where we use Neumann conditions on all three sides of the domain representing the region above the photosphere. We do not find bifurcating solutions. Instead high-energy solutions with detached field lines evolve smoothly from low energy solutions which have all field lines attached to the photosphere. Again the presence or absence of detached flux is dependent on the magnitude of $\lambda$ for those fields which are evolved quasi-statically via an increase in the plasma pressure.

In many studies the quasi-static evolution of the magnetic field and the associated plasma has been investigated for bipolar arcade structure. In this thesis we present a number of new results for multiple arcade structures. For multiple arcades we do not find bifurcations, and the consequent multiple solutions, as the field is evolved through a sequence of equilibrium states, in contrast to the findings for single arcades. Further

Received 2nd November, 1998

Thesis submitted to the University of Newcastle, February 1998. Degree approved, August 1998. Supervisor: Dr Warren Wood.

Copyright Clearance Centre, Inc. Serial-fee code: 0004-9729/99 \$A2.00+0.00. 
we show that particular polarity arrangements within a pair of arcade structures lead to quite different topologies of the field as it is evolved. When new flux emerges from the photospheric boundary under a pre-existing magnetic arcade the results suggest that such a mechanism will initiate coronal mass ejection. The multiple source models are shown to have characteristics which are in better agreement with new observations than the classical bipolar models.

We present new theoretical models of an atmospheric arcade with a quadrupolar source term. In this investigation we show that a magnetic hammock or dip arises provided the strengths of the inner and outer arcades differ. Such a dip is thought to be a region where plasma may be supported in the solar atmosphere. We show also that the topology of the atmospheric field is strongly influenced by the presence of shear and the temperature of the isothermal atmosphere. An important conclusion from these model calculations is that the basic topology of the magnetic structure is unaffected by them extent of the computational domain.

The analysis of the stability of a selection of these equilibrium models is performed using a normal mode analysis. The aim of this analysis is to examine the normal mode spectrum of a model atmosphere with a curved two dimensional magnetic field present. The atmosphere's response to a small perturbation from its equilibrium structure leads to an understanding of the possible behaviour changes these magnetic structures may undergo in the coronal region of the sun. The range of eigenvalues for each model atmosphere shows the richness in the oscillatory behaviour of these structures, while other modes show the possible growth/decay rates of the structures. These modes may lead to instabilities in the atmospheric magnetic fields. Further, it is shown that for these model calculations the normal mode spectra are strongly dependent on the basic topology of the equilibrium magnetic field. These results are commented on with respect to the presence or absence of neutral lines in the magnetic topology and their implication to the validity of quasi-static evolution of these structures.

Department of Mathematics The University of Newcastle Callaghan NSW 2308

Australia 